\title{
The Role of Imaging and Navigation for Natural Orifice Translumenal Endoscopic Surgery
}

\author{
Jens Rassweiler, M.D., ${ }^{1}$ Matthias Baumhauer, Ph.D., ${ }^{2}$ Uwe Weickert, M.D., ${ }^{3}$ Hans-Peter Meinzer, Ph.D., ${ }^{2}$ \\ Dogu Teber, M.D., ${ }^{1}$ Li-Ming Su, M.D., ${ }^{4}$ and Vipul R. Patel, M.D. ${ }^{5}$
}

\begin{abstract}
Background and Purpose: One of the next frontiers of minimally invasive surgery is natural orifice translumenal endoscopic surgery (NOTES). This article focuses on the perspectives and limitations of imaging and navigation in NOTES soft-tissue surgery.

Materials and Methods: Based on our in-vitro and in-vivo studies, with the applicability of different systems for image-guided soft-tissue endoscopic surgery, early experience with NOTES, and long-term experience with advanced endoluminal, laparoscopic robot-assisted endoscopic surgery, we performed a review of the literature. The aim was a critical analysis of the current role of imaging during NOTES.

Results: There are several steps/problems with NOTES that might be significantly alleviated by use of imaging and soft-tissue navigation. One has to distinguish between preoperative planning and intraoperative imaging or navigation. NOTES represents a hybrid technique of laparoscopy and endoscopy with similar limitations in perception and two-dimensional imaging; however, the use of flexible instruments increases the complexity with respect to the spatial orientation. This applies not only for the surgeon, but also for tracking devices for surgical navigation systems. Unlike optical navigation systems, electromagnetic and endoscopic (inside-out) tracking devices might be best suited to NOTES.

Conclusion: The safe realization and standardization of NOTES represents a real challenge that necessitates close and effective interdisciplinary collaboration of surgeon, technicians, informatics, and endoscopic and applied industries. Image-guided soft-tissue navigation may be very helpful to minimize the hazards of the technically challenging procedure.
\end{abstract}

\section{Introduction}

T $\mathrm{N}$ THE LAST TWO DECADES, open surgery has been challenged and revolutionized by various minimally invasive techniques (Table 1). This was based on technologic progress in the application of physical energy (ie. extracorporeal shockwave lithotripsy [SWL], laser), ${ }^{1-5}$ the improvement of video technology (ie, three-chip charge-coupled device cameras, chip on the tip) ${ }^{6-8}$ the introduction of robots or robotic manipulators (ie, da Vinci Surgical System, Intuitive Surgical, Sunnyvale, CA), ${ }^{9,10}$ and significant improvement of imaging techniques (ie, ultrasonography, CT, MRI). ${ }^{11-14}$

Even for highly standardized procedures, such as retrograde intrarenal surgery (RIRS) or laparoscopic radical prostatectomy, there are some limitations. ${ }^{15,16}$ Both techniques have realized adequate access techniques (ie, access sheath to the upper ureter, balloon dissection of the extraperitoneal space), but there is still limited maneuverability of the instruments and a significant loss of haptic sense:

- During RIRS, the spatial orientation might be difficult despite the use of intraoperative fluoroscopy, and in the case of caliceal stones, direct treatment is hampered because of the restricted flexibility of the laser fiber. Therefore, such stones have to be entrapped in a tipless basket and placed into the pelvis. ${ }^{17}$

- During laparoscopy, endoscopic suturing still necessitates significant expertise because of the small working space and the limited degrees of freedom (DOF) (ie, four DOF) of the instruments (ie, needle driver). ${ }^{18,19}$

\footnotetext{
${ }^{1}$ Department of Urology, SLK Kliniken, University of Heidelberg, Heilbronn, Germany.

${ }^{2}$ Division of Medical and Biological Informatics, German Cancer Research Center, Heidelberg, Germany.

${ }^{3}$ Department of Gastroenterology, SLK Kliniken, University of Heidelberg, Heilbronn, Germany.

${ }^{4}$ Robotic and Minimally Invasive Urologic Surgery, Department of Urology, University of Florida, College of Medicine, Gainsville, Florida.

${ }^{5}$ Global Robotics Institute, Florida Hospital Celebration Health, University of Central Florida, Orlando, Florida.
} 
Table 1. Role of Imaging and Technology in the Development of Minimally Invasive Techniques in Endourology

\begin{tabular}{lll}
\hline Procedure & \multicolumn{1}{c}{ Technical aspects } & \multicolumn{1}{c}{ Imaging } \\
\hline Extracorporeal shockwave lithotripsy & Shockwave source & Fluoroscopy/ultrasonography \\
High focused ultrasound (HIFU) energy & HIFU source & Transrectal ultrasonography \\
Percutaneous nephrolithotomy & Video technology & Fluoroscopy/ultrasonography \\
& Endoscopes & CT preoperatively (complex stones) \\
& Access devices & \\
Lithotripters (laser, ultrasound) & Fluoroscopy. CT preoperatively \\
& Video technology & Virtual reality trainer \\
& Endoscopes (rigid/flexible) & \\
Instruments/device & (holmium laser, basket) & Preoperative imaging \\
Laparoscopy & Access sheath (RIRS) & Intraoperative ultrasonography \\
& Video technology & (only partial nephrectomy) \\
& Endoscopes & Soft-tissue navigation (?) \\
Robot-assisted laparoscopy & Trocars/ports & Preoperative imaging \\
& Video technology & Soft-tissue navigation (?) \\
& (3D) & Virtual reality trainer (?) \\
\end{tabular}

$\mathrm{CT}=$ computed tomography; RIRS = retrograde intrarenal surgery; $3 \mathrm{D}=$ three-dimensional.

- With the da Vinci-device, providing three-dimensional (3D) vision and six DOF instruments, some of these problems can be overcome, but there is still the lack of tactile feedback. ${ }^{20}$

Natural orifice translumenal endoscopic surgery (NOTES) represents a new technique that shares characteristics of laparoscopic and endoluminal surgery. ${ }^{21}$ Therefore, the above-mentioned shortcomings are of major importance for safe transfer of this novel approach. We already have promising early experiences with the use of soft-tissue navigation for laparoscopic and robot-assisted surgery. ${ }^{22-24}$ These posi- tive experiences should be transferred to the application of NOTES.

We present an overview of the status of imaging and softtissue navigation with special emphasis on possible applications for NOTES.

\section{Materials and Methods}

Based on our in-vitro and in-vivo studies, with the applicability of different systems for image-guided soft-tissue endoscopic surgery, early experience with NOTES, and longterm experience with advanced endoluminal, laparoscopic

Table 2. Potential Problems of Clinical Introduction of NOtes (According to the Natural Orifice Surgery Consortium for Assessment and Research) and Possible Solution Provided by Imaging and Navigation

Problem Solution

Access to peritoneal cavity

Closure of entrance (ie, stomach, colon, bladder)

Prevention of infection

Development of multitasking platforms

Spatial orientation

Development of suturing and anastomotic devices Management of complications

Development of surgical steps Training
Preoperative imaging with 3D-reconstruction Intraoperative imaging (ie, ultrasonography) Specific suturing device (ie, Syntheon, Endostitch, Endoclinch)

Irrigation (ie, bladder), antibiotic prophylaxis, portals/platforms/sheaths with watertight access

Use of single $v s$ multiple platforms Preoperative imaging to determine the optimal access / placement of platform(s) = simulation

Preoperative imaging with 3D-reconstruction Intraoperative soft tissue navigation

Intraoperative imaging to control needle passage Intraoperative imaging for early diagnosis

Preoperative imaging and simulation of surgery

Development of virtual reality simulators based original imaging studies 
Table 3. Different Tracking Systems for Surgical Navigation ProceduresCOMparison Between Their Usability for Applications in LAPAROscopy and NOTES

\begin{tabular}{|c|c|c|}
\hline Tracking system & Laparoscopy & NOTES \\
\hline \multicolumn{3}{|l|}{ External systems } \\
\hline Optical tracking & $\begin{array}{l}\text { Adjusted to rigid instrument } \\
\text { Calibration necessary }\end{array}$ & Localization of flexible instrument problematic \\
\hline Electromagnetic tracking & $\begin{array}{l}\text { Mounted on flexible } \\
\text { ultrasound probe }\end{array}$ & $\begin{array}{l}\text { Localization of the flexible endoscope } \\
\text { Problems with interference of electric } \\
\text { devices (ie, HF-cautery) }\end{array}$ \\
\hline \multicolumn{3}{|l|}{ Internal systems } \\
\hline Endoscopic (inside-out) tracking & $\begin{array}{l}\text { Use of the endoscope placement } \\
\text { of navigation aids/definition } \\
\text { of anatomic landmarks }\end{array}$ & $\begin{array}{l}\text { Localization of structure by using the } \\
\text { endoscope after placement of markers / } \\
\text { according to well-defined anatomic structures }\end{array}$ \\
\hline
\end{tabular}

NOTES = natural orifice translumenal endoscopic surgery; HF = high frequency.

robot-assisted endoscopic surgery, we performed a review of the literature. The aim was a critical analysis of the current role of imaging during NOTES. We searched in wellestablished literature databases (Medline, PubMed) using the terms endoscopic navigation, soft-tissue navigation, augmented reality, image-guided surgery, and NOTES.

\section{Results}

The Natural Orifice Surgery Consortium for Assessment and Research (NOSCAR) identified several potential barriers to safe clinical implementation of NOTES (Table 2), including problems with access, spatial orientation, surgical performance (use of platforms, suturing), management of complications, and training. ${ }^{25}$ Several of these challenges may be addressed adequately by use of imaging-guided systems and soft-tissue navigation.

There are further obstacles related to the specific technique of NOTES, which are mainly related to the fact that flexible instruments and platforms are used. This may have a significant impact on the application of tracking systems (Table 3).
A

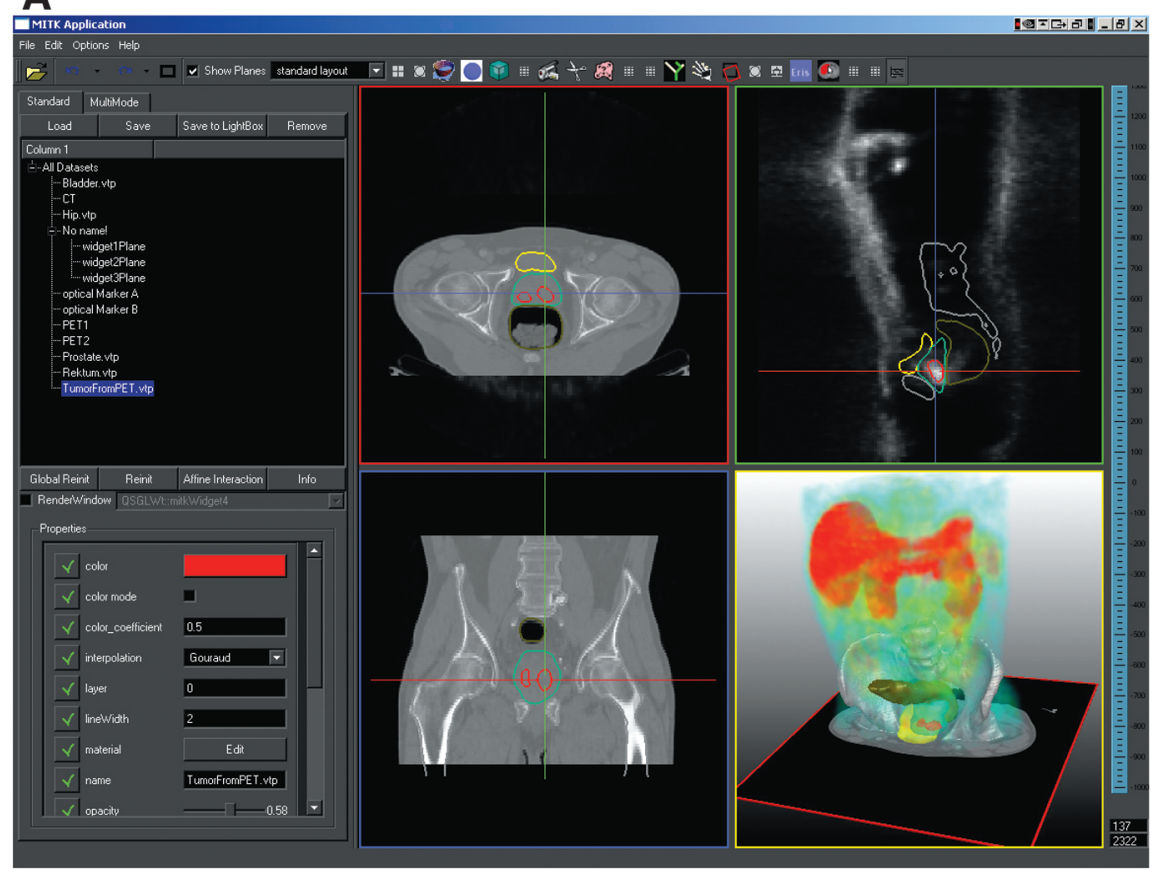

B

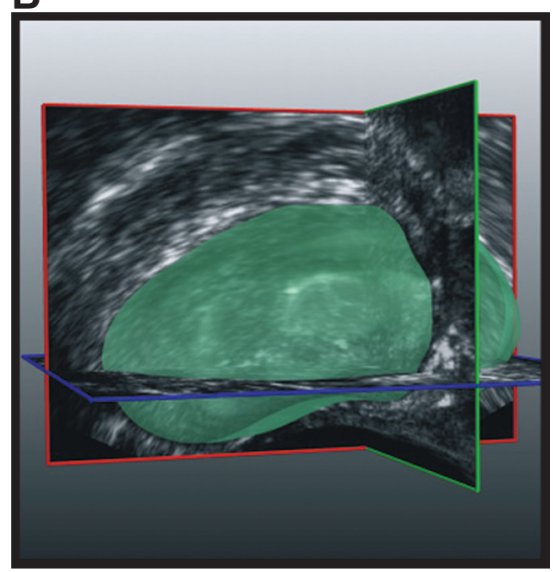

FIG. 1. Preoperative imaging using different imaging techniques with subsequent processing (segmentation). (A) PET/CT: Optimizing the visualization of the anatomic details by overlay of the different findings (here, two choline-PET-positive lesions in the prostate). In addition, the 3D-reconstruction improves the spatial orientation of the surgeon and may be useful to choose the site of the portals for NOTES (ie, transgastric access to the appendix or prostate). (B) 3D-transrectal ultrasonography (TRUS): Calculation of a 3D-prostate based on multiple slices using a stepper (ie, Pro Focus Expert; B\&K Medical, Quickborn, Germany) or using a 3D-TRUS scanner (ie, Voluson 760, GE, Solingen, Germany). Based on the data set, the shape of the prostate can be simulated even under conditions of deformation. PET=positron emission tomography; $\mathrm{CT}=$ computed tomography; $3 \mathrm{D}=$ three-dimensional; NOTES = natural orifice translumenal endoscopic surgery. 


\section{Preoperative imaging}

Medical imaging has played a role in the planning and guidance of surgical procedures since the advent of radiography (Table 1). Significant contributions to this field resulted from the incorporation of computers, creating 3D-images to depict pathoanatomic information. CT and MRI data can be recorded and displayed on the screen not only with the purpose of making an exact diagnosis, but also to augment the surgeon's spatial orientation (Fig. 1). As in intraluminal and laparoscopic surgery, imaging techniques play a similar role to assess the correct diagnosis and to provide maximal information for planning of any NOTES procedure.

\section{Image-guided systems}

Image-guided systems were initially adopted by the disciplines of neurosurgery and orthopedics for which they were well suited because of the fixed anatomy of these surgical fields (ie, head, brain, hip replacement). ${ }^{11,12}$ For urologic applications, mainly percutaneous or transrectal, ultrasonography and fluoroscopy were used to increase accuracy. Examples are puncture of the collecting system, stone localization for shockwave lithotripsy, and placement of seeds during brachytherapy (Table 1$).^{26,27}$

\section{Rigid tissue navigation}

The target structures are assumed to be undeformable and have a constant spatial relationship to anatomic landmarks. Before image acquisition, either bone or skin-applied navigation aids are attached near the anatomic regions of interest. A surgical planning step localized these navigation aids in the computerized imaging data (ie. CT, MRI). ${ }^{22}$ The step of postprocessing includes the definition of anatomic structures of interest providing important additional information, such as surgical trajectory, access, or security margins.

Immediately before surgery, the navigation system is set up in the operating room. During intraoperative patient-toimage registration, navigation aids are localized, and the transformation for mapping objects of the image to the tracking device space is calculated. This enables the visualization of arbitrary anatomic structures in relation to the surgical instruments. If possible, anatomic landmarks can be spotted in both medical image and tracking device data or the characteristic surface of organs or body parts (ie, kidney, face). Alternatively, imaging devices and surgical instruments can be tracked, so that intraoperatively both can be localized in the same coordinate frame simultaneously. A registration may not be necessary as long as the patient does not move.

Limitations of rigid tissue navigation. Each of these steps can be a potential source of error for overall navigation system accuracy. Errors of marker-based navigation systems for rigid anatomy can be divided into:

- Fiducial localization errors

- Fiducial registration errors

- Target registration errors (TRE)

TRE is the most important type of error, as it reflects the distance between an arbitrary point and its corresponding point in the registered space. ${ }^{22}$

\section{Soft-tissue navigation}

In soft-tissue navigation, however, all postimaging changes of surgical anatomy because of organ shift and tissue deformation add up directly to the resulting TRE and its associated target visualization error. Thus, whenever navigation targets are subject to considerable motion or deformation between preoperative imaging and intraoperative image-guidance, none of the established rigid navigation approaches will be sufficient for reliable soft-tissue navigation. This applies in particular to NOTES, except from transnasal approaches for intracranial surgery.

Steps of soft-tissue navigation. The main steps of imagingguided surgery include:

- Preoperative imaging

- Operative planning

- Intraoperative imaging

- Tracking

To illustrate the differences of the tracking systems with respect to their usefulness for NOTES, one has to focus on the tracking devices, organ shift, tissue deformation, and the user interface. Preoperative imaging usually will be based on thinsliced CT, MRI, or even PET/CT, MRI-spectroscopy (Fig. 1). Special software programs allow display of the data in $3 \mathrm{D}$ to fuse images of different modalities. The information from different modalities regarding the same anatomic region can then be displayed on one image. These images represent the basis for the planning of the procedure, but intraoperative image acquisition is mandatory for any surgical navigation.

Image acquisition. The most popular image modality used intraoperatively in soft-tissue navigation is intraoperative ultrasonography, either percutaneous (ie, percutaneous nephrolithotomy), transrectal (ie, high focused ultrasonography),

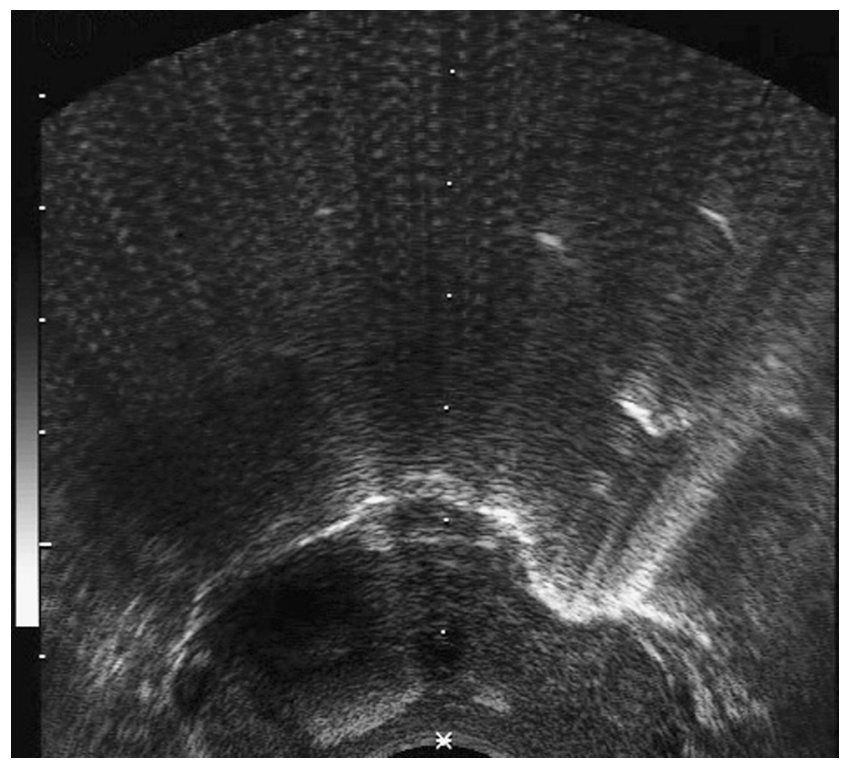

FIG. 2. Intraoperative imaging of the instrument (ie, Endo Dissect) pushing on the left lobe of the prostate with subsequent deformation of the gland. 
endoluminal (ie, endopyelotomy), or laparoscopic (ie, partial nephrectomy, cryotherapy, radiofrequency ablation). Most systems enable only two-dimensional (2D) images. ${ }^{28}$ In NOTES, endoluminal ultrasonography has been used successfully to control a balloon anastomosis device for enteroenteral anastomosis in the porcine model-to guide the placement of a suture when performing a fundoplication. ${ }^{29}$

Whereas in such situations, the imaging device (2D ultrasonography, 2D transrectal ultrasonography [TRUS], fluoroscopy) is used mainly manually to guide instruments or needles (Fig. 2), computed navigation requires 3D data. We have early experience with an approach for navigated laparoscopic radical prostatectomy based on the intraoperative processing of 3D transrectal ultrasound volumes. ${ }^{22}$ Apart from ultrasonography, open MRI or an isocentric 3D C-arm (like for SWL) may be useful for intraoperative image acquisition. $^{2}$
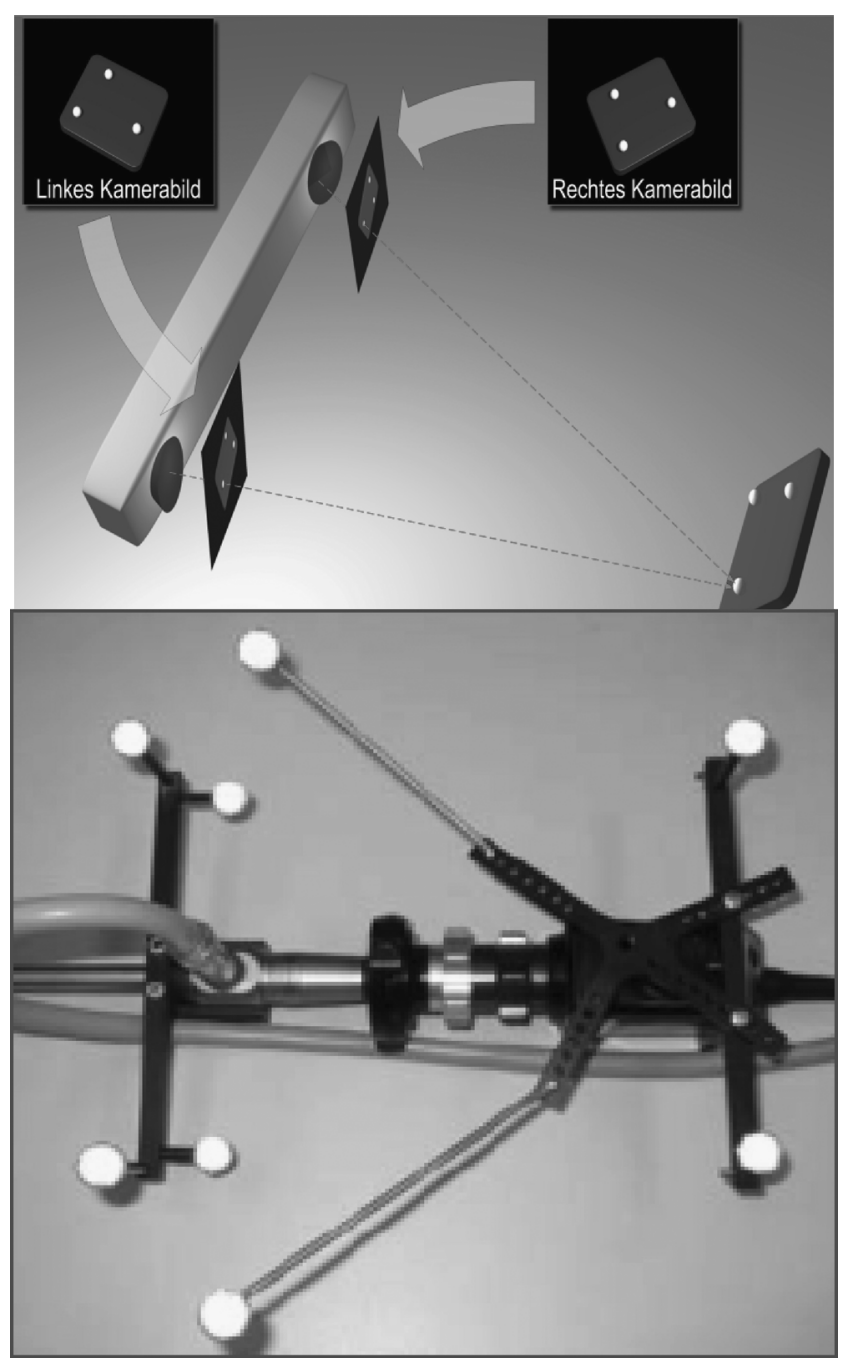

FIG. 3. Optical tracking system using two cameras mounted in the theater that register the signals from several antennas mounted on a rigid laparoscope or instrument. Problematic application for NOTES, because of the flexibility of the endoscope and instruments.
Image registration. A major challenge of soft-tissue navigation represents the registration of preoperative image data and intraoperative patient images, because the constellation and shape of unconstrained organs may change since planning and also during the intervention. In the case of NOTES, this may be of minor importance if anatomically clearly defined structures are approached, such as the gallbladder or the appendix; however, a retrocecal localization of the appendix may be problematic. Perhaps the decisive point might be if the structure of interest can easily be identified optically after (safe) entrance of the peritoneal cavity. A nonrigid image-toimage registration of preoperative and intraoperative image data may resolve the issue.

The main problem lies in the identification of corresponding anatomic details of surfaces. A surface-based method for correlation of preoperative liver images and laparoscopic 2D ultrasonography was superior to a point-based registration (error 2.9 vs $5 \mathrm{~mm}$ ). Marescaux and associates ${ }^{30}$ superimposed a 3D-visualization of the kidney and adrenal glands on the endoscopic image with manual overlapping of the organ boundaries. Accuracy is limited, however, because the 3Dreconstruction and the endoscopic image are displayed on a 2D-screen.

This really is the main problem of overlapping techniques: Ukimura and colleagues $^{31}$ superimposed the course of the neurovascular bundle imaged by duplex-TRUS on a 2Dendoscopic image. Surgeons cannot really estimate the third dimension. The same applies to our own experiences using inside-out tracking (Fig. 3). In laparoscopy, the integration of such images on the 3D-monitors of the da Vinci-system may overcome this problem; for NOTES, however, such 3Dendoscopic images are far away from any technical realization. Nevertheless, point-to-point registration may provide

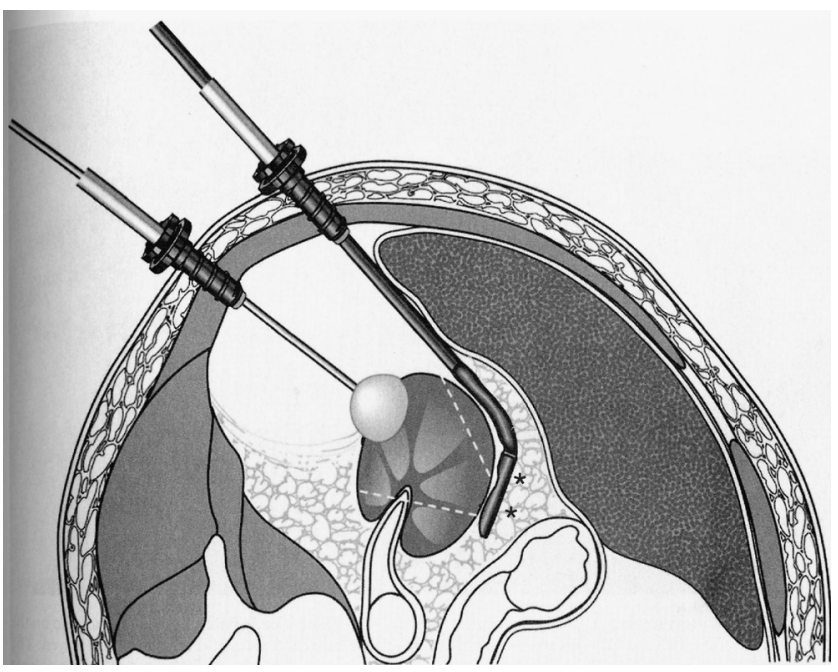

FIG. 4. Electromagnetic tracking of a laparoscopic ultrasound probe. Sensor coil is attached to the tip of the transducer (asterisks) to determine the position and orientation (POSE) of the B-scan plane during laparoscopic-assisted cryotherapy of a small renal tumor (adapted from Ukimura and Gill et al, 2007). Such a tracking technique would be applicable for NOTES (ie, determination of the POSE of the endoscope). 
optimal accuracy as long as the landmarks or fiducial markers can be spotted exactly in both image systems.

\section{Tracking devices}

Tracking devices link the spatial information from imaging modalities, surgical instruments, or the endoscope by representing them in a single coordinate system. There are several technical options:

- Optical tracking

- Electromagnetic tracking

- Magneto-optic tracking (hybrid tracking)

- Inside-out (endoscopic) tracking

Optical tracking. This is based on the visual registration (ie. by two cameras) of the position of tracking bodies or sensors attached to an endoscope, instrument, or ultrasound probe (Fig. 3). This determines the exact position of the tip of a rigid instrument/endoscope in the operating room inside the patient. For laparoscopy, this has been applied by Ukimura and associates ${ }^{31}$ and Ukimura and Gill ${ }^{32}$ during laparoscopic radical prostatectomy and partial nephrectomy. In the case of NOTES, this type of tracking system may be problematic, if not impossible, because all instruments and the endoscope have flexible tips; thus, the actual position and orientation of a tracking target cannot be captured by optical tracking.

Electromagnetic tracking. This measures the position of small coils inside the patient, without the need of direct line of sight. A magnetic field has to be generated around the patient. The sensor coil is attached to the tip of an instrument, endoscope, or ultrasound transducer. ${ }^{33,34}$ This technology already has been used with flexible ultrasound probes and would be applicable for NOTES (Fig. 4). Magnetic tracking,
A

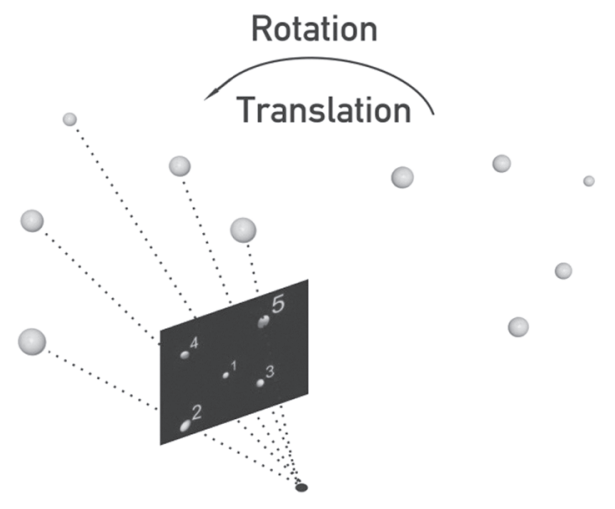

B
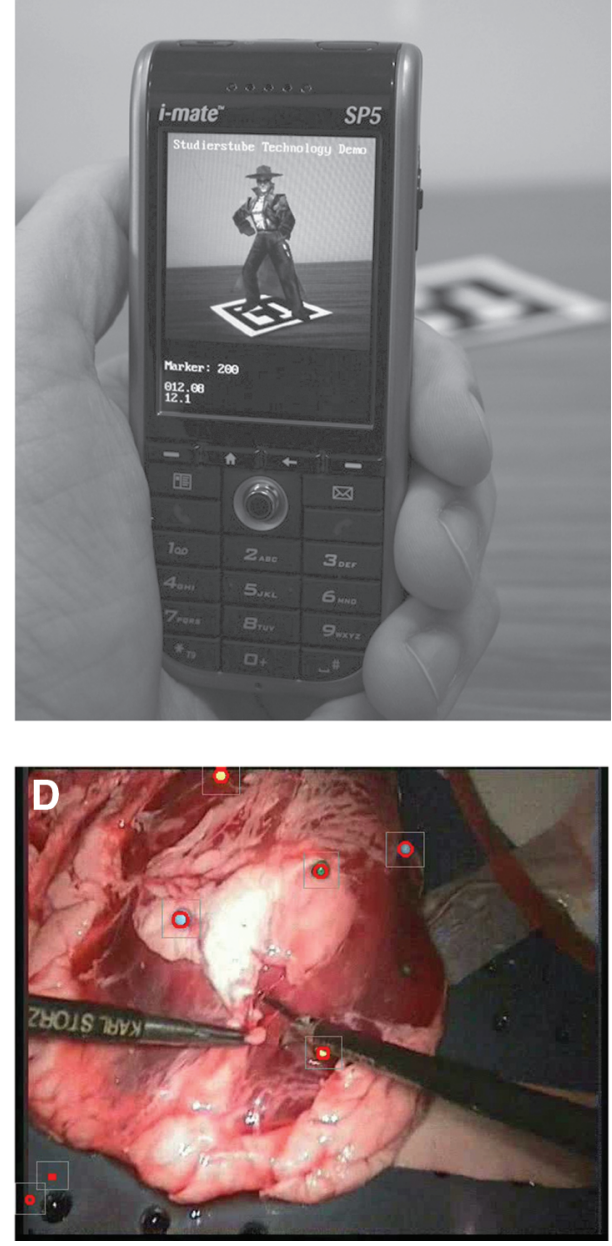

FIG. 5. Endoscopic tracking (inside-out) for intraoperative navigation. (A) The position and orientation of the endoscope (POSE) is calculated repeatedly on the basis of the distance to three to five markers (navigation aids). (B) The calculated POSE enables real-time reconstruction of a virtual image applicable for navigation (also used in video-cellular phone technology). (C) Experimental use in a perfused pelvitrainer model (P.O.P.-trainer, Optimist, Bregenz, Austria) with overlay of previously captured images (ie, retrograde pyelogram, renal angiography). Computerized adjustment of the virtual image based on the use of endoscopic tracking with six laparoscopically placed navigation aids. (D) Endoscopic image during real-time navigation. Such a system seems to be applicable for NOTES. 

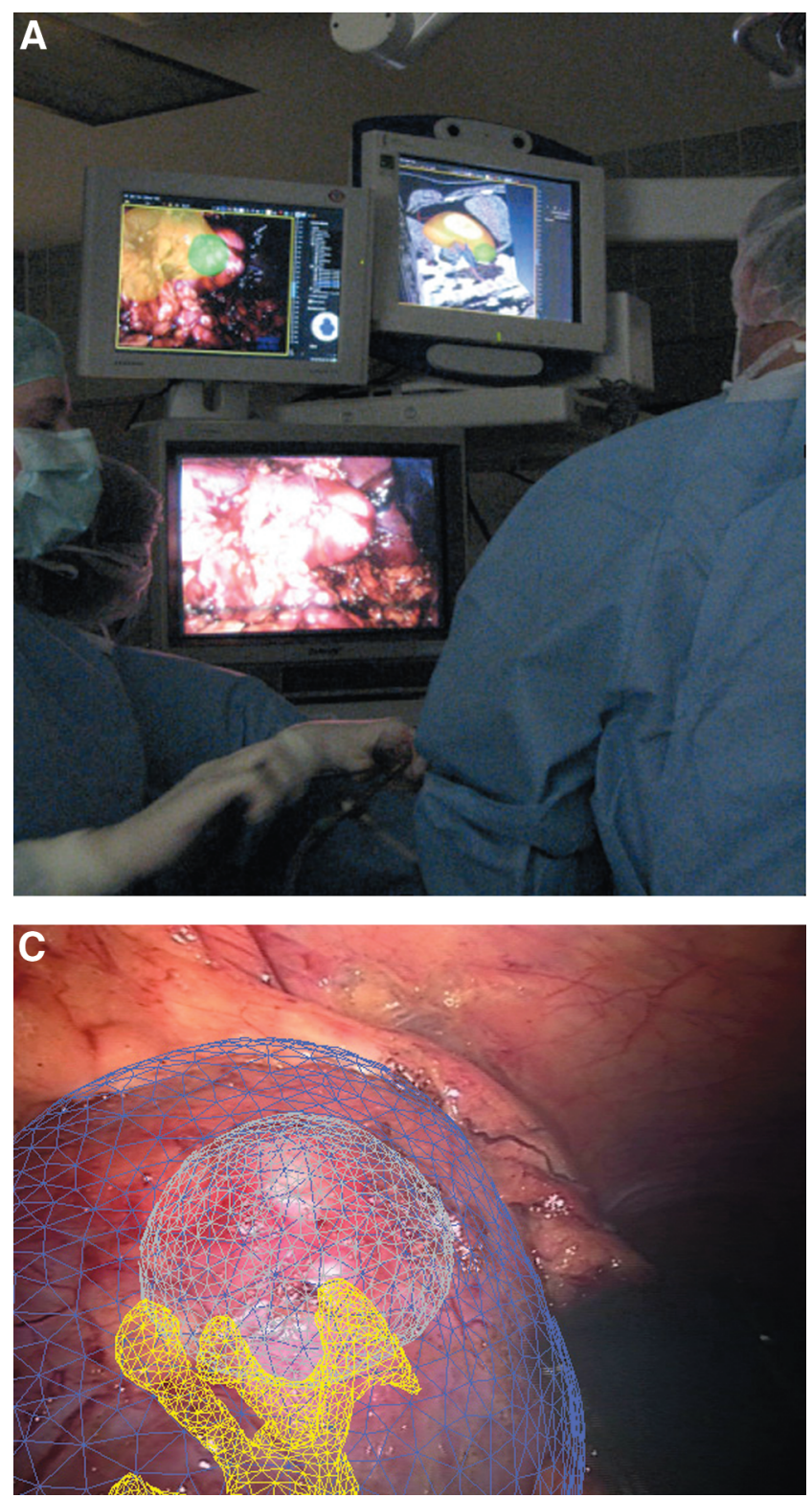

however, may be significantly interfered with by ferromagnetic objects like the surgical table, instruments, or electricpowered devices (ie. high-frequency generator). Such devices are absolutely necessary for any NOTES procedure. Accordingly, the combined use of optical and magnetic tracking devices will not be able to compensate for the aforementioned disadvantages.

Inside-out endoscopic tracking. This uses the information of the monocular video for tracking (Fig. 5). Based on the placement of navigation aids for the definition of anatomic landmarks, the position and orientation of the tip of the endoscope in relation to the target organ can be calculated. Theoretically, this approach should be ideal for use during NOTES, because it is independent of the degree of deflection of the instrument. Moreover, it can be integrated easily in the operative setting (Fig. 6A).

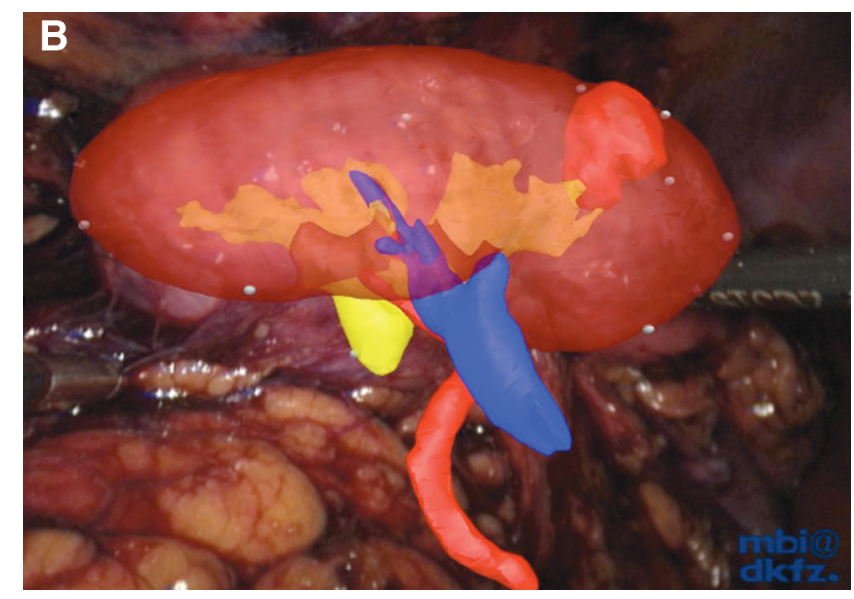

FIG. 6. Clinical application of endoscopic tracking during laparoscopic and robot-assisted surgery. (A) Setting of the different monitors during laparoscopic partial nephrectomy. (B) Manual overlay of the virtual image on the endoscopic picture. (C) Semiautomated fusion of the shape of the kidney based on the segmented data of the preoperative CT-scan with the endoscopic image (adapted from Vagvolgyi et $\mathrm{al}^{23}$ ). CT $=$ computed tomography.

There might be the problem, however, in placing the navigation aids adequately, which can be performed much more easily laparoscopically. A possible solution could be the endoscopic identification of anatomic landmarks (ie, lower pole of the kidney) together with a manual overlay (fusion) of the virtual on the endoscopic picture (Fig. 6B,C). ${ }^{23,24}$ Again, in such a setting, the 2D/3D-visualization problem has to be solved, particularly with respect to the relatively small optical systems used for NOTES.

\section{Computer-assisted colonoscopy}

To facilitate colonoscopy, a new computer-assisted colonoscope (The NeoGuide Colonoscopy System, NeoGuide Systems, San Jose, CA) has been developed recently and used in a small series of patients. ${ }^{35}$ The new colonoscope has added a tip position sensor inside the scope to measure the tip 
steering commands of the endoscopist and an external position sensor to measure the colonoscope's insertion depth. The system also contains additional control elements in multiple segments behind the scope tip. Each segment is the same basic length as the tip segment itself. These added elements are electromechanically controlled. By combining a measurement of the scope's depth, the tip position at each depth, and these computer-controlled segments, the NeoGuide Endoscopy System actively articulates the scope behind the tip, following the natural shape of the colon. The system offers the ability to display a real-time 3D map of the colon and the position of the colonoscope. The diameter of the endoscope $(14-20 \mathrm{~mm})$, however, hampers its use for NOTES, and the accuracy of the tracking systems is still limited.

\section{Organ shift and tissue deformation}

Continuous intraoperative navigation may overcome the constraints of organ shift and tissue deformation during surgery. It necessitates a constant correction (ie, recalculation), however, of the recorded data for reliable accuracy. This might be realized by the application of soft-tissue modeling methods, such as spline-based mathematical approaches or by use of biomechanical models. ${ }^{36}$ These models could also be used for compensation of motion caused by heartbeat and respiration. Since NOTES is mainly related to soft-tissue surgery, such modeling methods are very important. One of the main issues of navigation would be to minimize the amount of dissection to identify the relevant anatomic structure.

\section{User interface}

Concerning preoperative imaging and planning, 3Dvisualization is mandatory, but the segmentation of the data can offer more features, such as volume or surface rendering (Fig. 6C). Like in standard laparoscopy, such data are very helpful when the anatomic structure (ie, renal tumor, renal cyst) cannot be directly identified. The same would apply to NOTES, particularly in the case of urologic indications. Theoretically, these images can be directly superimposed on the video image (Fig. 6B); practically, however, this is limited by the fact that usually a 2D-videoimage is used and the superimposed image (=augmented reality) may disturb the view of anatomical details.

Based on this, we prefer a separate screen for visualization of navigation systems rather than the image-in-image technique. ${ }^{24}$ In the case of NOTES, these problems seem to be even more pronounced. The instuments have to travel a rather long, sometimes curved distance to the object (I,. transvaginally, transgastric, transvesical). ${ }^{37-41}$ Of course, this could be partially compensated for by the use of platforms or access sheaths; however, the dissection has to be performed in a relatively small working field in $2 \mathrm{D}$. We anticipate that the use of image-guided systems would be ideal to navigate the instruments easily to the target and probably to minimize the dissection, but the display of this information should not overload the endoscopic video screen.

\section{Discussion}

Of the aforementioned barriers to safe clinical implementation of NOTES, the following might be significantly alleviated by the use of image guidance:

\section{Preoperative planning of access to the intra- or extraperitoneum}

Initially, the transgastric route represented the classic access for NOTES to the peritoneum. In the meantime, several different orifices have been used experimentally and clinically, including the vagina, bladder, umbilicus, and even the colon. Moreover, one may also speculate about an extra- or retroperitoneal access, particularly for urologic indications. The site of any viscerotomy must be chosen with care to avoid blood vessels, adjacent viscera, and to have an optimal straight access to the target organ for the intended procedure.

Until now, introperative imaging has been restricted to percutaneous ultrasonography or even only transillumination of the abdominal wall. Another option was the additional use of small caliber laparoscopes (ie, needlescope) to provide insufflation of $\mathrm{CO}_{2}$ and optical control of the viscerotomy (ie, stomach, vagina, bladder). ${ }^{36-40}$ Endoscopic navigation should be able to visualize for the surgeon the exact position of the instrument/endoscope in relationship to other organs. Moreover, in the ase of more complex operations that need a doubleaccess technique (ie, transgastric plus transvesical), the optimal position and orientation of the two sites can be simulated and intraoperatively calculated. Of course, there are several restrictions; for example, the movement of bowel depending on the positioning of the patient (ie, supine vs Trendelenburg) might be difficult to assess - to be simulated by tissue modeling models.

\section{Spatial orientation}

NOTES is a hybrid technique that uses flexible instruments and endoscopes and represents a new challenge even for experienced gastroenterologists or laparoscopists. Understanding the orientation of the endoscope tip is one of the main difficulties. Theoretically, endourologists who are using both techniques for different indications in the upper and lower urinary tract (ie, flexible cystoscopy, flexible ureterorenoscopy, RIRS, laparoscopy, retroperitoneoscopy) should have a shorter learning curve. With the transgastric access, several viscera lie in a retrograde position (ie, gallbladder, spleen, liver). Visualization obtained with a retroflexed scope may result in an inverted off-axis image. Real-time tracking of the endoscope tip with the ability to display the location and orientation of the scope would be a significant advantage during NOTES.

\section{Identification of anatomy and pathology}

In contrast to the laparoscopic approach, NOTES is limited by the number of access channels and the dexterity of the instruments. In the case of intra-abdominal organs, such as gallbladder or appendix, identification of the target might be relatively easy, and image guidance may not be needed. In the case of intraparenchymal lesions (ie, renal cyst, renal tumor) that are covered by tissue (ie, perirenal fat) and in the case of retroperitoneal organs such as the adrenals, navigation might be extremely helpful. 2,29,30,33,34 Augmented reality allows visualization of organs covered by tissue, thereby enabling straight and minimally traumatic access.

\section{Perspectives}

Use of NOTES potentially represents a new era for intraand retroperitoneal surgery. While NOSCAR identified 
potential barriers to safe clinical implementation of this hybrid technique, rapid technologic advances are addressing these issues. Based on the significant contribution of rigid navigation for procedures in orthopedic, maxillofacial, and neurosurgery, the early applications of new imaging systems for soft-tissue navigation are promising. The use of flexible instruments and the limitation to 2D-endoscopes may also restrict the applicability of some of these techniques (ie, optical tracking). There is no doubt, however, that image guidance will soon play a role in video-surgical procedures, and NOTES will benefit directly from this technical progress.

\section{Conclusion}

The safe realization and standardization of NOTES represents a real challenge that necessitates close and effective interdisciplinary collaboration of surgeon, technicians, informatics, and endoscopic and applied industries. Image-guided soft-tissue navigation may be very helpful to minimize the hazards of the technically challenging procedure.

\section{Disclosure Statement}

No competing financial interests exist.

\section{References}

1. Chaussy C, Schmiedt E, Jocham D, Brendel W, Forssmann B, Walther V. First clinical experience with extracorporeally induced destruction of kidney stones by shock waves. J Urol 1982;127: 417-420.

2. Rassweiler J, Henkel TO, Köhrmann KU, Potempa D, Jünemann KP, Alken P. Lithotripter technology: Present and future. J Endourol 1992;6:1-13.

3. De la Rosette JJ, D'Ancona FC, Debruyne FM. Current status of thermotherapy of the prostate. J Urol 1997;157:430 438.

4. Köhrmann KU, Michel MS, Rassweiler JJ, Alken P. (1996) Shock waves Focused ultrasound. In: Smith A, Badlani G, Bagley D, Clayman RV, Jordan G, Kavoussi LR, Lingeman JE, Preminger GM, Segura JW, eds. Smith's Textbook of Endourology. St. Louis, Mo: Quality Medical, 1996, pp 661-679.

5. Chaussy CG, Thüroff $S$. High-intensive focused ultrasound in localized prostate cancer. J Endourol 2000;14:293-299.

6. Rassweiler J, Frede T, Seemann O, Stock C, Sentker L. Telesurgical laparoscopic radical prostatectomy. Initial experience. Eur Urol 2001:40:75-83.

7. Janetschek G, Rassweiler J. Future outlook. In: Janetschek G, Rassweiler J, Griffith D, eds. Laparoscopic Surgery in Urology. New York: Thieme Stuttgart, 1996, pp 276-280.

8. Fuchs GJ. Milestones in endoscopic design fpr minimally invasive urologic surgery: The sentinel role of a pioneer. Surg Endosc 2006;20(suppl 2):S493-499.

9. Rassweiler J, Binder J, Frede T. Robotic and telesurgery: Will they change our future? Curr Opin Urol 2001;11:309-320.

10. Lirici MM, Papaspyropoulos V, Angelini L. Telerobotics in medicine and surgery. Min Invas Ther Allied Technol 1997;6:364-378.

11. Paul HA, Bargar WL, Mittelstadt B, et al. Development of a surgical robot for cementless hip arthoplasty. Clin Orthop Relat Res1992;285:57-66.

12. Nimsky C, Ganslandt O, Kober H, Moller M. Ulmer S, Tomandi B, Fahlbusch R. Integration of functional magnetic resonance imaging supported by magnetoencephalography in functional neuronavigation. Neurosurgery 1999;44:12491256.

13. Klimek L, Ecke U, Lübben B, Witte J, Mann W. A passivemarker-based optical system for computer-aided surgery in otorhinolaryngology: Development and first clinical experiences. Laryngoscope 1999;109:1509-1515.

14. Su LM, Stoianovici D, Jarrett TW, et al. Robotic percutaneous access to the kidney: Comparison with standard manual access J Endourol 2002;16:471-475.

15. Chung SY, Chon CH, Ng CS, Fuchs GJ. Simultaneous bilateral retrograde intrarenal surgery for stone disease in patients with significant comorbidities. J Endourol 2006;20: 761-765.

16. Rassweiler J, Schulze M, Teber D, Seemann O, Frede T. Laparoscopic radical prostatectomy: Functional and oncological outcomes. Curr Opin Urol 2004;14:75-82.

17. Fuchs GJ, Yurkanin GP. Endoscopic surgery for renal calculi. Curr Opin Urol 2003;13:243-247.

18. Frede T, Stock C, Renner C, Budair Z, Abdel-Salam Y, Rassweiler J. Geometry of laparoscopic suturing and knotting techniques. J. Endourol 1999;13:191-198.

19. Rassweiler J, Marrero R, Hammady A, Erdogru T, Teber D, Frede T. Transperitoneal laparoscopic radical prostatectomy: Ascending technique. J. Endourol 2004;18:593-600.

20. Patel VR, Tully AS, Holmes R, Lindsay J. Robotic radical prostatectomy in the community setting-the learning curve and beyond: Initial 200 cases. J Urol 2005;174:269-272.

21. Swain P. A justification for NOTES-natural orifice translumenal endosurgery. Gastointest Endosc 2007;65:514-516.

22. Baumhauer M, Feuerstein M, Meinzer HP, Rassweiler J. Navigation in endoscopic soft tissue surgery-perspectives and limitations. J Endourol 2008;22:751-766.

23. Vagvolgyi B, Reiley CE, Hager GD, Taylor R, Levinson AW, $\mathrm{Su}$ L-M. Toward direct registration of video to computer tomography for intraoperative surgical planning during laparoscopic partial nephrectomy J Endourol 2007;21 (suppl):A25. Abstract BR6-12.

24. Teber D, Baumhauer M, Simpfendörfer T, Hruza M, Klein J, Schulze M, Rassweiler J. Augmented reality a new tool to improve surgical accuracy during laparoscopic partial nephrectomy J. Endourol 2007;21(suppl):A72. Abstract MP09-20.

25. Rattner D, Kalloo A; ASGE/SAGES Working Group. ASGE/ SAGES working group on Natural Orifice Translumenal Endoscopic Surgery. October 2005. Surg Endosc 2006;20:329-333.

26. Pisani E, Montanari E, Deiana G, Trincheri A, Zanetti G, Tzoumas S. Robotized prostate biopsy. Minimally Invas Ther 1995;4:289-291.

27. Bove P, Stoianovici D, Micali S, Patriciu A, Grassi N, Jarrett TW, Vespasiani G, Kavoussi LR. Is telesurgery a new reality? Our experience with laparoscopic and percutaneous procedures. J Endourol 2003;17:137-142.

28. Frede T, Hatzinger M, Rassweiler J. Ultrasound in endourology. J Endourol 2001;15:3-16.

29. Swain P. Endoscopic suturing: Now and incoming. Gastrointest Endosc Clin N Am 2007;17:505-520.

30. Marescaux J, Rubino F, Arenas M, Mutter D, Sohen L. Augmented-reality-assisted laparoscopic adrenalectomy. JAMA 2004;292:2214-2215.

31. Ukimura O, Magi-Galluzzi C, Gill IS. Real-time transrectal ultrasound guidance during laparoscopic radical prostatectomy: Impact on surgical margins. J Urol 2006;175:13041310.

32. Ukimura O, Gill IS. Imaging-assisted endoscopic surgery: Cleveland Clinic experience. J Endourol 2008;22:803-810. 
33. Kindratenko VV. A survey of electromagnetic position tracker calibration techniques. Virtual reality: Research, development, and applications. 2000;5:169-182.

34. Feuerstein M, Reichl T, Vogel J, Schneider A, Feussner H, Navab N. Magneto-optic tracking of a flexible laparoscopic ultrasound transducer for laparoscope augmentation. Med Image Comput Assist Interv Int Conf. 2007;10:458-466.

35. Eickhoff A, van Dam J, Jakobs R, et al. Computer-assisted colonoscopy (the NeoGuide Endoscopic System): Results of the first human clinical trial ("PACE study"). Am J Gastroenterol 2007;102:261-266.

36. Carter TJ, Sermesant M, Cash DM, Barratt DC, Tanner C, Hawkes DJ. Application of soft tissue modelling to imageguided surgery. Med Eng Phys 2005;27:893-909.

37. Rolanda C, Lima E, Pego JM, et al. Third-generation cholecystectomy by natural orifices: Transgastric and transvesical combined approach (with video). Gastrointest Endosc 2007: 65:111-117.

38. Lima E Rolanda C, Osorio L, Pego J, Henriques-Coelho T, Silva D, Carvalho J, Correia-Pinto J. Scarless nephrectomy by NOTES. J Endourol 2007;21(suppl). Abstract MP 13-1.

39. Marescaux J, Dallemagne B, Perretta S, Wattiez A, Mutter D. Surgery without scars: Report of transluminal cholecystectomy in a human being. Arch Surg 2007;142:823-827.

40. Gettman MT, Lotan Y, Napper CA, Cadeddu JA. Transvaginal laparoscopic nephrectomy: Development and feasibility in the porcine model. Urology 2002:59:446-450.

41. Swain P. Nephrectomy and natural orifice translumenal endosurgery (NOTES): Transvaginal, transgastric, transrec- tal, and transvesical approaches. J Endourol 2008;22: 811-818.

Address reprint requests to: Jens Rassweiler, M.D. Department of Urology SLK Kliniken Heilbronn Am Gesundbrunnen 20 D-74074 Heilbronn Germany E-mail: jens.rassweiler@slk-kliniken.de

$\quad$ Abbreviations Used
$\mathrm{CT}=$ computed tomography
DOF $=$ degrees of freedom
$\mathrm{MRI}=$ magnetic resonance imaging
NOSCAR $=$ Natural Orifice Surgery Consortium for
$\quad$ Assessment and Research
NOTES $=$ natural orifice translumenal endoscopic surgery
PET $=$ positron emission tomography
SWL $=$ shockwave lithotripsy
RIRS $=$ retrograde intrarenal surgery
3D $=$ three-dimensional
$2 \mathrm{D}=$ two-dimensional
TRE $=$ target registration errors
TRUS $=$ transrectal ultrasonography

